\title{
SUMMABILITY OF DOUBLE SEQUENCES BY WEIGHTED MEAN METHODS AND TAUBERIAN CONDITIONS FOR CONVERGENCE IN PRINGSHEIM'S SENSE
}

\author{
FERENC MÓRICZ and U. STADTMÜLLER
}

Received 16 March 2004

\begin{abstract}
After a brief summary of Tauberian conditions for ordinary sequences of numbers, we consider summability of double sequences of real or complex numbers by weighted mean methods which are not necessarily products of related weighted mean methods in one variable. Our goal is to obtain Tauberian conditions under which convergence of a double sequence follows from its summability, where convergence is understood in Pringsheim's sense. In the case of double sequences of real numbers, we present necessary and sufficient Tauberian conditions, which are so-called one-sided conditions. Corollaries allow these Tauberian conditions to be replaced by Schmidt-type slow decrease conditions. For double sequences of complex numbers, we present necessary and sufficient so-called two-sided Tauberian conditions. In particular, these conditions are satisfied if the summable double sequence is slowly oscillating.
\end{abstract}

2000 Mathematics Subject Classification: 40E05, 40B05, 40 G05.

1. Introduction. We begin with a brief and concise summary of the corresponding well-known results for single sequences. For basic facts on summability theory, we refer to $[4,9,12]$ for ordinary sequences and to [1] for double sequences.

Let $p=\left(p_{k}: k=0,1,2, \ldots\right)$ be a fixed sequence of nonnegative numbers with $p_{0}>0$, and set

$$
P_{m}:=\sum_{k=0}^{m} p_{k}, \quad k=0,1, \ldots
$$

Weighted means of a sequence $\left(s_{k}: k=0,1, \ldots\right)$ of complex numbers are defined by

$$
t_{m}:=\frac{1}{P_{m}} \sum_{k=0}^{m} p_{k} s_{k}, \quad m=0,1, \ldots
$$

The sequence $\left(s_{k}\right)$ is said to be summable by the weighted mean method determined by the sequence $p$, in short, summable $(\bar{N}, p)$, if the sequence $\left(t_{m}\right)$ converges to a finite limit $s$; in symbols, $s_{m} \rightarrow s(\bar{N}, p)$.

The summability method $(\bar{N}, p)$ is said to be regular, if $s_{m} \rightarrow s$ implies $s_{m} \rightarrow s(\bar{N}, p)$. It is well known that $(\bar{N}, p)$ is regular if and only if $P_{m} \rightarrow \infty$ as $m \rightarrow \infty$ (see, e.g., [9, page $16])$, which we assume in the following. 
We are interested in converse conclusions. In general, $s_{m} \rightarrow s(\bar{N}, p)$ implies $s_{m} \rightarrow s$ only under additional so-called Tauberian conditions. Set

$$
a_{k}:=s_{k}-s_{k-1}, \quad k=0,1, \ldots ; s_{-1}:=0 .
$$

Then, each of the following conditions is Tauberian for the method $(\bar{N}, p)$ :

$$
\begin{gathered}
\sum_{k=1}^{m} a_{k} P_{k-1}=o\left(P_{m}\right), \\
\sum_{k=1}^{\infty}\left|a_{k}\right|^{2} \frac{P_{k-1}}{p_{k-1}}<\infty, \quad p_{k}>0 \forall k>0, \\
a_{k}=o\left(\frac{p_{k-1}}{P_{k-1}}\right),
\end{gathered}
$$

since they yield $t_{m}-s_{m} \rightarrow 0$ as $m \rightarrow \infty$. The $o$-type condition (1.6) can be weakened to an $O$-type condition

$$
a_{k}=O\left(\frac{p_{k-1}}{P_{k-1}}\right)
$$

or even to a one-sided condition

$$
a_{k} \leq c \frac{p_{k-1}}{P_{k-1}},
$$

where $c$ is a positive constant and in the last case we suppose that $p$ also satisfies the condition $p_{m} / P_{m} \rightarrow 0$ as $m \rightarrow \infty$.

A necessary and sufficient Tauberian condition was given in [8], which is implied by either of the conditions (1.4)-(1.8). To present it, we recall the following two definitions. Let $\lambda=(\lambda(m))$, where $\lambda(m)>m$ for all $m$, be an increasing sequence of natural numbers such that

$$
\liminf _{m \rightarrow \infty} \frac{P_{\lambda(m)}}{P_{m}}>1
$$

and denote by $\Lambda_{u}$ the set of all such sequences $\lambda$. Similarly, let $\mu=(\mu(m))$, where $\mu(m)<m$ for all $m$, be a nondecreasing sequence of natural numbers such that

$$
\liminf _{m \rightarrow \infty} \frac{P_{m}}{P_{\mu(m)}}>1
$$

and denote by $\Lambda_{\ell}$ the set of all such sequences $\mu$.

Now, the following theorem was proved in [8]: for a given sequence $\left(s_{m}\right)$ of real numbers, $s_{m} \rightarrow s(\bar{N}, p)$ implies $s_{m} \rightarrow s$ if and only if

$$
\begin{gathered}
\inf _{\lambda \in \Lambda_{u}} \limsup _{m \rightarrow \infty} \frac{1}{P_{\lambda(m)}-P_{m}} \sum_{k=m+1}^{\lambda(m)} p_{k}\left(s_{k}-s_{m}\right) \leq 0, \\
\inf _{\mu \in \Lambda_{\ell}} \limsup _{m \rightarrow \infty} \frac{1}{P_{m}-P_{\mu(m)}} \sum_{k=\mu(m)+1}^{m} p_{k}\left(s_{m}-s_{k}\right) \leq 0 .
\end{gathered}
$$


In case of smooth weights, for example, when

$$
p_{k}:=(k+1)^{\alpha}, \quad k=0,1, \ldots ; \alpha>-1,
$$

we may replace $\Lambda_{u}$ by $\tilde{\Lambda}_{u}:=\{([\lambda m]): \lambda>1\}$ and $\Lambda_{\ell}$ by $\tilde{\Lambda}_{\ell}:=\{([m / \lambda]): \lambda>1\}$, where [a] denotes the integer part of $a>0$. Furthermore, either the condition of slow decrease in the sense of Schmidt [10] (see also [4, pages 124-125])

$$
\lim _{\lambda \rightarrow 1+} \liminf _{m \rightarrow \infty} \min _{m<k \leq \lambda m}\left(s_{k}-s_{m}\right) \geq 0,
$$

or the condition of slow increase

$$
\lim _{\lambda \rightarrow 1+} \limsup _{m \rightarrow \infty} \max _{m<k \leq \lambda m}\left(s_{k}-s_{m}\right) \leq 0,
$$

implies both (1.11a) and (1.11b) (see [8] for details).

In the same paper, the following theorem for complex sequences was proved: $s_{m} \rightarrow$ $s(\bar{N}, p)$ implies $s_{m} \rightarrow s$ if and only if one of the following Tauberian conditions is satisfied:

$$
\inf _{\lambda \in \Lambda_{u}} \limsup _{m \rightarrow \infty}\left|\frac{1}{P_{\lambda_{m}}-P_{m}} \sum_{k=m+1}^{\lambda(m)} p_{k}\left(s_{k}-s_{m}\right)\right|=0
$$

or

$$
\inf _{\mu \in \Lambda_{\ell}} \limsup _{m \rightarrow \infty}\left|\frac{1}{P_{m}-P_{\mu(m)}} \sum_{k=\mu(m)+1}^{m} p_{k}\left(s_{m}-s_{k}\right)\right|=0 .
$$

The following special case is called the condition of slow oscillation (see, e.g., [6]):

$$
\inf _{\lambda \in \Lambda_{u}} \limsup _{m \rightarrow \infty} \max _{m<k \leq \lambda(m)}\left|s_{k}-s_{m}\right|=0 .
$$

In this case (1.15a) (and a fortiori, (1.15b)) is clearly satisfied. The symmetric counterpart involving the class $\Lambda_{\ell}$ can be formulated analogously.

From now on, we will consider double sequences. Let $p=\left(p_{k, \ell}: k, \ell=0,1, \ldots\right)$ be a fixed double sequence of nonnegative numbers with $p_{0,0}>0$, and set

$$
P_{m, n}:=\sum_{k=0}^{m} \sum_{\ell=0}^{n} p_{k, \ell}, \quad m, n=0,1, \ldots
$$

The weighted means of a double sequence $\left(s_{k, \ell}: k, \ell=0,1, \ldots\right)$ of complex numbers are defined by

$$
t_{m, n}:=\frac{1}{P_{m, n}} \sum_{k=0}^{m} \sum_{\ell=0}^{n} p_{k, \ell} s_{k, \ell}, \quad m, n=0,1, \ldots
$$

The sequence $\left(s_{k, \ell}\right)$ is said to be summable by the weighted mean method determined by the sequence $p$, in short, summable $(\bar{N}, p)$, if the double sequence $\left(t_{m, n}\right)$ converges 
to a finite number $s$ in Pringsheim's sense; in symbols, $s_{m, n} \rightarrow s(\bar{N}, p)$. This type of convergence means that for every $\varepsilon>0$ there exists a natural number $n_{0}(\varepsilon)$ such that

$$
\left|t_{m, n}-s\right|<\varepsilon \quad \forall m, n \geq n_{0}(\varepsilon) .
$$

Furthermore, we also consider the so-called bounded convergence (in Pringsheim's sense); in symbols, $b-\lim s_{m, n}=s$, which means the following:

$$
s_{m, n} \longrightarrow s, \quad\left|s_{m, n}\right| \leq K \quad \forall m, n=0,1, \ldots,
$$

where $K$ is a finite positive number. Similarly, $b-\lim s_{m, n}=s(\bar{N}, p)$ means that $b-$ $\lim t_{m, n}=s$.

It is known (see, e.g., [3]) that

$$
b-\lim s_{m, n}=s \Longrightarrow b-\lim s_{m, n}=s(\bar{N}, p)
$$

if and only if

$$
\lim _{m, n \rightarrow \infty} \frac{P_{k, n}}{P_{m, n}}=0 \quad \forall k
$$

and

$$
\lim _{m, n \rightarrow \infty} \frac{P_{m, \ell}}{P_{m, n}}=0 \quad \forall \ell .
$$

In particular, it follows from (1.22) that $P_{m, n} \rightarrow \infty$ as $m, n \rightarrow \infty$.

Of particular interest are the weighted means of multiplicative structure, that is, when $p_{k, \ell}=p_{k} q_{\ell}$, where $\left(p_{k}\right)$ and $\left(q_{\ell}\right)$ are sequences of nonnegative real numbers with $p_{0}, q_{0}>0$. Given a double sequence $\left(s_{m, n}\right)$, we set

$$
a_{m, n}:=s_{m, n}-s_{m-1, n}-s_{m, n-1}+s_{m-1, n-1}, \quad m, n=0,1, \ldots,
$$

with the agreement that $s_{m, n}=0$ if $m<0$ or $n<0$.

It is known (see [11]) that if

$$
P_{m}:=\sum_{k=0}^{m} p_{k} \longrightarrow \infty, \quad Q_{n}:=\sum_{\ell=0}^{n} q_{\ell} \longrightarrow \infty \quad m, n \longrightarrow \infty,
$$

then the pair of conditions

$$
\sup _{n \geq 0}\left|\sum_{\ell=0}^{n} a_{m, \ell}\right| \leq c \frac{p_{m-1}}{P_{m-1}} \quad \forall m, \quad \sup _{m \geq 0}\left|\sum_{k=0}^{m} a_{k, n}\right| \leq c \frac{q_{n-1}}{Q_{n-1}} \quad \forall n,
$$

where $c$ is some constant, is a Tauberian condition; that is, under conditions (1.25) and (1.26), $\lim s_{m, n}=s(\bar{N}, p)$ implies $\lim s_{m, n}=s$. We observe that (1.26) can be considered to be a multiplicative version of the Tauberian condition (1.7). However, $a_{m, n}$ $=O\left(p_{m-1} q_{n-1} / P_{m-1} Q_{n-1}\right)$ (compare (1.7)) is not a Tauberian condition, as it has been shown in [5] for the $(C, 1,1)$-mean. 
2. Main results. In the sequel we will need the following notations. Let $m, n, \mu, v$ be nonnegative integers and set

$$
a_{\mu}(n):=\sum_{\nu=0}^{n} a_{\mu, \nu}, \quad b_{\nu}(m):=\sum_{\mu=0}^{m} a_{\mu, \nu},
$$

where $a_{\mu, v}$ is defined in (1.24). Clearly, we have

$$
a_{\mu}(n):=s_{\mu, n}-s_{\mu-1, n}, \quad b_{v}(m):=s_{m, v}-s_{m, v-1} .
$$

Furthermore, set

$$
p_{k}(n):=\sum_{\nu=0}^{n} p_{k, v}, \quad q_{\ell}(m):=\sum_{\mu=0}^{m} p_{\mu, \ell}, \quad k, \ell=0,1, \ldots
$$

Now, the multivariate version of the Tauberian condition (1.4) reads as follows.

THEOREM 2.1. If $s_{m, n} \rightarrow s(\bar{N}, p)$ and one of the following Tauberian conditions is satisfied,

$$
\sum_{\mu=1}^{n} P_{\mu-1, n} \max _{\ell \leq n}\left|a_{\mu}(\ell)\right|+\sum_{v=1}^{n} P_{m, v-1}\left|b_{v}(m)\right|=o\left(P_{m, n}\right)
$$

or

$$
\sum_{\mu=1}^{n} P_{\mu-1, n}\left|a_{\mu}(n)\right|+\sum_{v=1}^{n} P_{m, v-1} \max _{k \leq m}\left|b_{v}(k)\right|=o\left(P_{m, n}\right),
$$

then $s_{m, n} \rightarrow s$.

The multivariate version of Tauberian condition (1.5) is more involved.

THEOREM 2.2. Assume that (1.22) holds. If $b-\lim s_{m, n}=s(\bar{N}, p)$ and one of the following Tauberian conditions is satisfied: for every $\varepsilon>0$ there exist natural numbers $\mu_{0}$, $v_{0}$ such that

$$
\begin{gathered}
\sum_{\mu=\mu_{0}}^{\infty} \frac{P_{\mu-1, n}}{p_{\mu-1}(n)} \max _{\ell \leq n}\left|a_{\mu}(\ell)\right|^{2}<\varepsilon \quad \text { for large } n, \\
\sum_{v=v_{0}}^{\infty} \frac{P_{m, v-1}}{q_{v-1}(m)}\left|b_{v}(m)\right|^{2}<\varepsilon \quad \text { for large } m
\end{gathered}
$$

or

$$
\begin{gathered}
\sum_{\mu=\mu_{0}}^{\infty} \frac{P_{\mu-1, n}}{p_{\mu-1}(n)}\left|a_{\mu}(n)\right|^{2}<\varepsilon \quad \text { for large } n, \\
\sum_{v=v_{0}}^{\infty} \frac{P_{m, v-1}}{q_{v-1}(m)} \max _{k \leq m}\left|b_{v}(k)\right|^{2}<\varepsilon \quad \text { for large } m,
\end{gathered}
$$

then $b-\lim s_{m, n}=s$. 
Now, we turn to our main result which provides a necessary and sufficient Tauberian condition to deduce the conclusion $s_{m, n} \rightarrow s$ from $s_{m, n} \rightarrow s(\bar{N}, p)$. A result of this type was already discussed in [7] for the $(C, 1,1)$-mean and for more general weighted means in [11], however, the assumptions there can be simplified and the proof in the present paper is direct.

To formulate Theorem 2.5, we introduce the notation

$$
\triangle(m, n ; k, \ell):=\sum_{m<\mu \leq k} \sum_{n<v \leq \ell} p_{\mu, v}=P_{k, \ell}-P_{k, n}-P_{m, \ell}+P_{m, n},
$$

where $k>m$ and $\ell>n$. We consider a pair of nondecreasing sequences $\left(\lambda_{1}(m)\right.$ $\left.>m, \lambda_{2}(n)>n: m, n=1,2, \ldots\right)$ of natural numbers such that

$$
\begin{gathered}
\liminf _{m, n \rightarrow \infty} \frac{P_{\lambda_{1}(m), \lambda_{2}(n)}}{P_{m, n}}>1, \\
\limsup _{m, n \rightarrow \infty} \frac{P_{\lambda_{1}(m), \lambda_{2}(n)}}{\triangle\left(m, n ; \lambda_{1}(m), \lambda_{2}(n)\right)}<\infty,
\end{gathered}
$$

and denote by $\Lambda_{u}$ the set of all such pairs of sequences.

Furthermore, we consider a pair of nondecreasing sequences $\left(\mu_{1}(m)<m, \mu_{2}(n)<\right.$ $n$ ) of natural numbers such that

$$
\begin{gathered}
\liminf _{m, n \rightarrow \infty} \frac{P_{m, n}}{P_{\mu_{1}(m), \mu_{2}(n)}}>1, \\
\limsup _{m, n \rightarrow \infty} \frac{P_{m, n}}{\triangle\left(\mu_{1}(m), \mu_{2}(n) ; m, n\right)}<\infty,
\end{gathered}
$$

and denote by $\Lambda_{\ell}$ the set of all such pairs of sequences.

We remind the reader that the limit infimum of a double sequence of real numbers is defined by

$$
\liminf _{m, n \rightarrow \infty} b_{m, n}:=\lim _{N \rightarrow \infty} \inf _{m, n \geq N} b_{m, n}
$$

where the right-hand side may be $-\infty$ or $\infty$. Obviously,

$$
\limsup _{m, n \rightarrow \infty} b_{m, n}=-\liminf _{m, n \rightarrow \infty}\left(-b_{m, n}\right) .
$$

EXAMPLE 2.3. Put $p_{m, n}=m+n$, then we have

$$
P_{m, n}=\left(\frac{1}{2}\right) m(m+1) n+\left(\frac{1}{2}\right) n(n+1) m \sim\left(\frac{1}{2}\right) n m(n+m),
$$

and a typical example of a sequence from $\Lambda_{u}$ is $\lambda_{1}(m)=[\lambda m], \lambda_{2}(n)=[\mu n]$ with $\lambda$ and $\mu>1$ leading to

$$
\triangle\left(m, n ; \lambda_{1}(m), \lambda_{2}(n)\right) \sim\left(\frac{1}{2}\right) n m\left(m\left(\lambda^{2} \mu-\lambda^{2}-\mu+1\right)+n\left(\mu^{2} \lambda-\mu^{2}-\lambda+1\right)\right) .
$$

We cannot expect that (2.8) is always satisfied, since for example with $p_{k, \ell}=e^{-k \ell}$ we have $P_{m, n}=n+m+o(1)$. However, in this case (1.22) is not satisfied. 
LEMMA 2.4. Under (1.22), the sets $\Lambda_{u}$ and $\Lambda_{\ell}$ are not empty.

Proof. Given $m_{0}, n_{0}$, we can find by (1.22) integers $m \geq m_{0}, n \geq n_{0}$ large enough such that $P_{m, n} \geq 3 P_{m_{0}, n}$ and $P_{m, n} \geq 3 P_{m, n_{0}}$ which implies that $\triangle\left(m_{0}, n_{0}, m, n\right) \geq$ (1/3) $P_{m, n}$.

The next result contains a multivariate version of the Tauberian conditions in (1.11).

THEOREM 2.5. Assume that the sets $\Lambda_{u}$ and $\Lambda_{\ell}$ are nonempty and $s_{m, n}$ is a double sequence of real numbers. Then, $s_{m, n} \rightarrow s(\bar{N}, p)$ implies $s_{m, n} \rightarrow s$ if and only if both of the following conditions are satisfied:

$$
\begin{aligned}
& \inf _{\Lambda_{u}} \limsup _{m, n \rightarrow \infty} \frac{1}{\triangle\left(m, n: \lambda_{1}(m), \lambda_{2}(n)\right)} \sum_{m<k \leq \lambda_{1}(m)} \sum_{n<\ell \leq \lambda_{2}(n)} p_{k, \ell}\left(s_{k, \ell}-s_{m, n}\right) \leq 0, \\
& \inf _{\Lambda_{\ell}} \limsup _{m, n \rightarrow \infty} \frac{1}{\triangle\left(\mu_{1}(m), \mu_{2}(n) ; m, n\right)} \sum_{\mu_{1}(m)<k \leq m} \sum_{\mu_{2}(n)<\ell \leq n} p_{k, \ell}\left(s_{m, n}-s_{k, \ell}\right) \leq 0 .
\end{aligned}
$$

REMARKS 2.6. (i) The conditions in (2.14) are obviously satisfied if

$$
\begin{aligned}
& \inf _{\Lambda_{u}} \limsup _{m, n \rightarrow \infty} \max _{\substack{m<k \leq \lambda_{1}(m) \\
n<\ell \leq \lambda_{2}(n)}}\left(s_{k, \ell}-s_{m, n}\right) \leq 0, \\
& \inf _{\Lambda_{\ell}} \limsup _{m, n \rightarrow \infty} \max _{\substack{\mu_{1}(m)<k \leq m \\
\mu_{2}(n)<\ell \leq n}}\left(s_{m, n}-s_{k, \ell}\right) \leq 0 .
\end{aligned}
$$

(ii) In the special (but important) case of multiplicative weights $p_{k, \ell}=p_{k} q_{\ell}$ with sequences $p, q$ as before, conditions (2.7) and (2.8) are satisfied if the sequences $\left(\lambda_{1}(m)\right)$ and $\left(\lambda_{2}(n)\right)$ of natural numbers are chosen such that

$$
\liminf _{m \rightarrow \infty} \frac{P_{\lambda_{1}(m)}}{P_{m}}>1, \quad \liminf _{n \rightarrow \infty} \frac{Q_{\lambda_{2}(n)}}{Q_{n}}>1
$$

Then, condition (2.7) is obviously satisfied. In addition, we have

$$
\frac{P_{\lambda_{1}(m), \lambda_{2}(n)}}{\triangle\left(m, n ; \lambda_{1}(m), \lambda_{2}(n)\right)}=\left\{\left(1-\frac{P_{m}}{P_{\lambda_{1}(m)}}\right)\left(1-\frac{Q_{n}}{Q_{\lambda_{2}(n)}}\right)\right\}^{-1},
$$

whence it follows that

$$
\begin{aligned}
& \limsup _{m, n \rightarrow \infty} \frac{P_{\lambda_{1}(m), \lambda_{2}(n)}}{\triangle\left(m, n ; \lambda_{1}(m), \lambda_{2}(n)\right)} \\
& \quad=\left\{\left(1-\limsup _{m \rightarrow \infty} \frac{P_{m}}{P_{\lambda_{1}(m)}}\right)\left(1-\limsup _{n \rightarrow \infty} \frac{Q_{n}}{Q_{\lambda_{2}(n)}}\right)\right\}^{-1}<\infty,
\end{aligned}
$$

which means that this time (2.8) is automatically satisfied.

(iii) In the special case when

$$
\lim _{m \rightarrow \infty} \frac{P_{m+1}}{P_{m}}=1, \quad \lim _{n \rightarrow \infty} \frac{Q_{n+1}}{Q_{n}}=1,
$$


we may replace (2.14a) by

$$
\inf _{\rho>1} \limsup _{m, n \rightarrow \infty} \frac{1}{\left(P_{m(\rho)}-P_{m}\right)\left(Q_{n(\rho)}-Q_{n}\right)} \sum_{m<k \leq m(\rho)} \sum_{n<\ell \leq n(\rho)} p_{k} q_{\ell}\left(s_{k, \ell}-s_{m, n}\right) \leq 0,
$$

where

$$
m(\rho):=\min \left\{k>m: \frac{P_{k}}{P_{m}} \geq \rho\right\}
$$

and $n(\rho)$ is defined analogously based on $\left(Q_{n}\right)$. A similar reformulation of condition (2.14b) reads as follows:

$$
\inf _{0<\sigma<1} \limsup _{m, n \rightarrow \infty} \frac{1}{\left(P_{m}-P_{m(\sigma)}\right)\left(Q_{n}-Q_{n(\sigma)}\right)} \sum_{m(\sigma)<k \leq m} \sum_{n(\sigma)<\ell \leq n} p_{k} q_{\ell}\left(s_{m, n}-s_{k, \ell}\right) \leq 0,
$$

where

$$
m(\sigma):=\max \left\{k<m: \frac{P_{k}}{P_{m}} \leq \sigma\right\}
$$

and $n(\sigma)$ is defined analogously based on $\left(Q_{n}\right)$.

(iv) It is clear that (2.20) and (2.22) are implied by the following conditions of slow increase:

$$
\begin{aligned}
& \lim _{\rho \rightarrow 1+} \limsup _{m, n \rightarrow \infty} \max _{\substack{m<k \leq m(\rho) \\
n<\ell \leq n(\rho)}}\left(s_{k, \ell}-s_{m, n}\right) \leq 0, \\
& \lim _{\sigma \rightarrow 1-} \limsup _{m, n \rightarrow \infty} \max _{\substack{m(\sigma)<k \leq m \\
n(\sigma)<\ell \leq n}}\left(s_{m, n}-s_{k, \ell}\right) \leq 0,
\end{aligned}
$$

respectively. Now, one can verify that the last two conditions are equivalent. Furthermore, all particular cases discussed in [11] can be deduced from (2.24a).

Finally, the counterpart of Theorem 2.5 when $\left(s_{m, n}\right)$ is a double sequence of complex numbers reads as follows.

THEOREM 2.7. Assume that the sets $\Lambda_{u}$ and $\Lambda_{\ell}$ are nonempty. Then, $s_{m, n} \rightarrow s(\bar{N}, p)$ implies $s_{m, n} \rightarrow s$ if and only if one of the following conditions is satisfied:

$$
\inf _{\Lambda_{u}} \limsup _{m, n \rightarrow \infty}\left|\frac{1}{\triangle\left(m, n ; \lambda_{1}(m), \lambda_{2}(n)\right)} \sum_{m<k \leq \lambda_{1}(m)} \sum_{n<\ell \leq \lambda_{2}(n)} p_{k, \ell}\left(s_{k, \ell}-s_{m, n}\right)\right|=0
$$


or

$$
\inf _{\Lambda_{\ell}} \limsup _{m, n \rightarrow \infty}\left|\frac{1}{\triangle\left(\mu_{1}(m), \mu_{2}(n) ; m, n\right)} \sum_{\mu_{1}(m)<k \leq m} \sum_{\mu_{2}(n)<\ell \leq n} p_{k, \ell}\left(s_{m, n}-s_{k, \ell}\right)\right|=0 .
$$

REMARK 2.8. The following special case may be called the condition of slow oscillation. If

$$
\inf _{\Lambda_{u}} \limsup _{m, n \rightarrow \infty} \max _{\substack{m<k \leq \lambda_{1}(m) \\ n<\ell \leq \lambda_{2}(n)}}\left|s_{k, \ell}-s_{m, n}\right|=0,
$$

then condition (2.25a) is obviously satisfied. The symmetric counterpart of (2.27) from which (2.26a) follows can be formulated analogously.

Originally, these conditions were considered in the case of multiplicative weights in $[2,11]$.

\section{Proofs}

Proof of Theorem 2.1. It hinges on the following decomposition. By (2.1), we may write

$$
\begin{aligned}
& \left|P_{m, n}\left(s_{m, n}-t_{m, n}\right)\right| \\
& =\left|\sum_{k=0}^{m} \sum_{\ell=0}^{n} p_{k, \ell}\left(s_{m, n}-s_{k, \ell}\right)\right| \\
& =\left|\sum_{k=0}^{m} \sum_{\ell=0}^{n} p_{k, \ell}\left\{\sum_{\mu=k+1}^{m} \sum_{v=0}^{\ell} a_{\mu, v}+\sum_{\mu=0}^{m} \sum_{v=\ell+1}^{n} a_{\mu, v}\right\}\right| \\
& =\left|\sum_{k=0}^{m} \sum_{\ell=0}^{n} p_{k, \ell}\left\{\sum_{\mu=k+1}^{m} a_{\mu}(\ell)+\sum_{v=\ell+1}^{n} b_{v}(m)\right\}\right| \\
& \leq \sum_{\mu=1}^{m} P_{\mu-1, n} \max _{1 \leq \ell \leq n}\left|a_{\mu}(\ell)\right|+\sum_{v=1}^{n} P_{m, v-1}\left|b_{v}(m)\right| .
\end{aligned}
$$

Thus, (2.4a) implies that $s_{m, n}-t_{m, n} \rightarrow 0$ as $m, n \rightarrow \infty$.

In the case of condition (2.4b), the above reasoning works if we use the decomposition

$$
\sum_{k=0}^{m} \sum_{\ell=0}^{n} p_{k, \ell}\left(s_{m, n}-s_{k, \ell}\right)=\sum_{k=0}^{m} \sum_{\ell=0}^{n} p_{k, \ell}\left\{\sum_{\mu=k+1}^{m} \sum_{\nu=0}^{n} a_{\mu, v}+\sum_{\mu=0}^{k} \sum_{\nu=\ell+1}^{n} a_{\mu, v}\right\} .
$$

Proof of Theorem 2.2. Assume that condition (2.5a) is satisfied. By the Cauchy inequality and (2.3), we have

$$
\begin{aligned}
& \sum_{\mu=1}^{m} P_{\mu-1, n} \max _{1 \leq \ell \leq n}\left|a_{\mu}(\ell)\right| \\
& \quad \leq\left\{\sum_{\mu=1}^{m} \frac{P_{\mu-1, n}}{p_{\mu-1}(n)} \max _{1 \leq \ell \leq n}\left|a_{\mu}(\ell)\right|^{2} \sum_{\mu=1}^{n} P_{\mu-1, n} p_{\mu-1}(n)\right\}^{1 / 2} \leq c P_{m, n},
\end{aligned}
$$


where $c$ is a constant. Similarly, we have

$$
\sum_{v=1}^{n} P_{m, v-1}\left|b_{v}(m)\right| \leq c P_{m, n}
$$

Taking into account (3.1) and the boundedness of the double sequence $\left(t_{m, n}\right)$, from the inequalities above we conclude that $\left(s_{m, n}\right)$ is also bounded, that is,

$$
\left|s_{m, n}\right| \leq K \quad \forall m, n=0,1, \ldots
$$

with some constant $K$. By (2.2), it follows that

$$
\left|a_{\mu}(\ell)\right| \leq 2 K, \quad\left|b_{v}(k)\right| \leq 2 K \quad \forall \mu, \nu, k, \ell=0,1, \ldots
$$

Now, we can proceed as follows. For any $\varepsilon>0$, choose natural numbers $\mu_{0}, v_{0}$ according to the assumptions in Theorem 2.2 and follow the estimations above to obtain

$$
\begin{aligned}
& \left\{\sum_{\mu=1}^{\mu_{0}-1}+\sum_{\mu=\mu_{0}}^{m}\right\} P_{\mu-1, n} \max _{1 \leq \ell \leq n}\left|a_{\mu}(\ell)\right| \\
& \quad \leq\left(\mu_{0}-1\right) P_{\mu_{0}-2, n} 2 K+\left\{\sum_{\mu=\mu_{0}}^{\infty} \frac{P_{\mu-1, n}}{p_{\mu}(n)} \max _{1 \leq \ell \leq n}\left|a_{\mu}(\ell)\right|^{2} \sum_{\mu=1}^{m} P_{\mu-1, n} p_{\mu-1}(n)\right\}^{1 / 2} \\
& \quad \leq P_{m, n}\left\{\left(\mu_{0}-1\right) 2 K \frac{P_{\mu_{0}-2, n}}{P_{m, n}}+\varepsilon^{1 / 2}\right\} \leq 2 P_{m, n} \varepsilon^{1 / 2},
\end{aligned}
$$

provided that $m, n$ are large enough (observe (1.22)). Similarly, we obtain

$$
\left\{\sum_{v=1}^{v_{0}-1}+\sum_{v=v_{0}}^{n}\right\} P_{m, v-1}\left|b_{v}(m)\right| \leq 2 P_{m, n} \varepsilon^{1 / 2}
$$

provided again that $m, n$ are large enough. Combining (3.1), (3.7), and (3.8) yields

$$
\left|s_{m, n}-t_{m, n}\right| \leq 4 \varepsilon^{1 / 2},
$$

which completes the proof since $\varepsilon$ was arbitrary. The proof is analogous if $(2.5 \mathrm{~b})$ is satisfied.

For the proof of Theorem 2.5, we need the following auxiliary result which is interesting in itself.

LEMMA 3.1. If $s_{m, n} \rightarrow s(\bar{N}, p)$, then for any pair of sequences $\left(\lambda_{1}(m)\right),\left(\lambda_{2}(n)\right) \in \Lambda_{u}$,

$$
\lim _{m, n \rightarrow \infty} \frac{1}{\triangle\left(m, n ; \lambda_{1}(m), \lambda_{2}(n)\right)} \sum_{m<k \leq \lambda_{1}(m)} \sum_{n<\ell \leq \lambda_{2}(n)} p_{k, \ell} s_{k, \ell}=s,
$$


and for any pair of sequences $\left(\mu_{1}(m)\right),\left(\mu_{2}(n)\right) \in \Lambda_{\ell}$,

$$
\lim _{m, n \rightarrow \infty} \frac{1}{\triangle\left(\mu_{1}(m), \mu_{2}(n) ; m, n\right)} \sum_{\mu_{1}(m)<k \leq m} \sum_{\mu_{2}(n)<\ell \leq n} p_{k, \ell} s_{k, \ell}=s .
$$

Proof. By definition, we may write that

$$
\begin{aligned}
& \quad \sum_{m<k \leq \lambda_{1}(m)} \sum_{n<\ell \leq \lambda_{2}(n)} p_{k, \ell} s_{k, \ell} \\
& \quad=P_{\lambda_{1}(m), \lambda_{2}(n)} t_{\lambda_{1}(m), \lambda_{2}(n)}-P_{\lambda_{1}(m), n} t_{\lambda_{1}(m), n}-P_{m, \lambda_{2}(n)} t_{m, \lambda_{2}(n)}+P_{m, n} t_{m, n} .
\end{aligned}
$$

Hence, a simple rearrangement gives

$$
\begin{aligned}
& \frac{1}{\triangle\left(m, n ; \lambda_{1}(m), \lambda_{2}(n)\right)} \sum_{m<k \leq \lambda_{1}(m)} \sum_{n<\ell \leq \lambda_{2}(n)} p_{k, \ell} s_{k, \ell} \\
& =t_{\lambda_{1}(m), \lambda_{2}(n)}+\frac{1}{\triangle\left(m, n ; \lambda_{1}(m), \lambda_{2}(n)\right)}\{ \\
& \quad\left(P_{\lambda_{1}(m), n}-P_{m, n}\right)\left(t_{\lambda_{1}(m), \lambda_{2}(n)}-t_{\lambda_{1}(m), n}\right) \\
& +\left(P_{m, \lambda_{2}(n)}-P_{m, n}\right)\left(t_{\lambda_{1}(m), \lambda_{2}(n)}-t_{m, \lambda_{2}(n)}\right) \\
& + \\
& \left.+P_{m, n}\left(t_{\lambda_{1}(m), \lambda_{2}(n)}-t_{\lambda_{1}(m), n}-t_{m, \lambda_{2}(n)}+t_{m, n}\right)\right\} .
\end{aligned}
$$

It remains to observe that by (2.6) we have

$$
P_{\lambda_{1}(m), n}-P_{m, n} \leq P_{\lambda_{1}(m), \lambda_{2}(n)}-\triangle\left(m, n ; \lambda_{1}(m), \lambda_{2}(n)\right),
$$

whence, by (2.8) we obtain

$$
\limsup _{m, n \rightarrow \infty} \frac{P_{\lambda_{1}(m), n}-P_{m, n}}{\triangle\left(m, n ; \lambda_{1}(m), \lambda_{2}(n)\right)}<\infty ;
$$

and similarly

$$
\limsup _{m, n \rightarrow \infty} \frac{P_{m, \lambda_{2}(n)}-P_{m, n}}{\triangle\left(m, n ; \lambda_{1}(m), \lambda_{2}(n)\right)}<\infty .
$$

Combining the last two inequalities with (3.13) and using the convergence of $\left(t_{m, n}\right)$, we conclude (3.10).

The proof of (3.11) is similar.

The following corollary is an immediate consequence of Lemma 3.1 by taking $\left(\lambda_{1}(m)\right.$, $\left.\lambda_{2}(n)\right)=(m+1, n+1)$.

\section{COROLLARY 3.2. If}

$$
\liminf _{m, n \rightarrow \infty} \frac{p_{m, n}}{P_{m, n}}>0
$$

then $s_{m, n} \rightarrow s(\bar{N}, p)$ if and only if $s_{m, n} \rightarrow s$. 
Proof of TheOrem 2.5. Necessity. It follows directly from Lemma 3.1 and the convergence of $\left(s_{m, n}\right)$. Even more is true, for every pair $\left(\lambda_{1}(m), \lambda_{2}(n)\right) \in \Lambda_{u}$, we have

$$
\lim _{m, n \rightarrow \infty} \frac{1}{\triangle\left(m, n ; \lambda_{1}(m), \lambda_{2}(n)\right)} \sum_{m<k \leq \lambda_{1}(m)} \sum_{n<\ell \leq \lambda_{2}(n)} p_{k, \ell}\left(s_{k, \ell}-s_{m, n}\right)=0 .
$$

An analogous limit relation holds for every pair $\left(\mu_{1}(m), \mu_{2}(n)\right) \in \Lambda_{\ell}$.

Sufficiency. Given $\varepsilon>0$, by (2.14a) there exists a pair $\left(\lambda_{1}(m), \lambda_{2}(n)\right) \in \Lambda_{u}$ such that

$$
\limsup _{m, n \rightarrow \infty} \frac{1}{\triangle\left(m, n ; \lambda_{1}(m), \lambda_{2}(n)\right)} \sum_{m<k \leq \lambda_{1}(m)} \sum_{n<\ell \leq \lambda_{2}(n)} p_{k, \ell}\left(s_{k, \ell}-s_{m, n}\right) \leq \varepsilon .
$$

By Lemma 3.1, we conclude that

$$
s-\liminf _{m, n \rightarrow \infty} s_{m, n} \leq \varepsilon
$$

In a similar way, by (2.14b) and Lemma 3.1, we conclude

$$
\limsup _{m, n \rightarrow \infty} s_{m, n}-s \leq \varepsilon
$$

Since $\varepsilon>0$ was arbitrary, (3.20) together with (3.21) yield $s_{m, n} \rightarrow s$ as $m, n \rightarrow \infty$.

Proof of Theorem 2.7. It is omitted since it follows similar arguments as the proof of Theorem 2.5 .

ACKNOWLEDGMENT. This research was started while the first author was visiting the University of Ulm, Germany, in October 2002, and was completed with the support of the Hungarian National Foundation for Scientific Research under Grant T 046192.

\section{REFERENCES}

[1] V. G. Chelidze, Summability Methods for Double Series and Double Integrals, Tbilisi University Press, Tbilisi, 1977.

[2] C.-P. Chen and J.-M. Hsu, Tauberian theorems for weighted means of double sequences, Anal. Math. 26 (2000), no. 4, 243-262.

[3] H. J. Hamilton, Transformations of multiple sequences, Duke Math. J. 2 (1936), 29-60.

[4] G. H. Hardy, Divergent Series, Clarendon Press, Oxford, 1949.

[5] K. Knopp, Limitierungs-Umkehrsätze für Doppelfolgen, Math. Z. 45 (1939), 573-589 (German).

[6] E. Landau, Über die Bedeutung einiger neuer Grenzwertsätze der Herren Hardy und Axel, Prace Mat. Fiz. 21 (1910), 97-177 (Polish).

[7] F. Móricz, Tauberian theorems for Cesàro summable double sequences, Studia Math. 110 (1994), no. 1, 83-96.

[8] F. Móricz and U. Stadtmüller, Necessary and sufficient conditions under which convergence follows from summability by weighted means, Int. J. Math. Math. Sci. 27 (2001), no. 7, 399-406.

[9] A. Peyerimhoff, Lectures on Summability, Lecture Notes in Mathematics, vol. 107, SpringerVerlag, Berlin, 1969.

[10] R. Schmidt, Über divergente Folgen und lineare Mittelbildungen, Math. Z. 22 (1925), 89-152 (German). 
[11] U. Stadtmüller, Tauberian theorems for weighted means of double sequences, Anal. Math. 25 (1999), no. 1, 57-68.

[12] K. Zeller and W. Beekmann, Theorie der Limitierungsverfahren, Ergebnisse der Mathematik und ihrer Grenzgebiete, vol. 15, Springer-Verlag, Berlin, 1970 (German).

Ferenc Móricz: Bolyai Institute, University of Szeged, Aradi vértanúk tere 1, 6720 Szeged, Hungary

E-mail address: moricz@math.u-szeged.hu

U. Stadtmüller: Abteilung Zahlentheorie und Wahrscheinlichkeitstheorie, Fakultät für Mathematik und Wirtschaftswissenschaften, Universität Ulm, 89069 Ulm, Germany

E-mail address: stamue@mathematik.uni-u $1 \mathrm{~m}$. de 


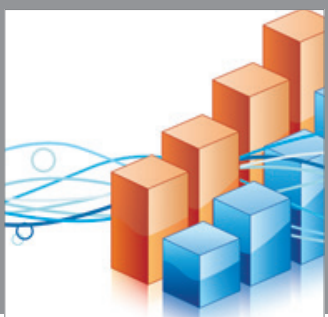

Advances in

Operations Research

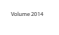

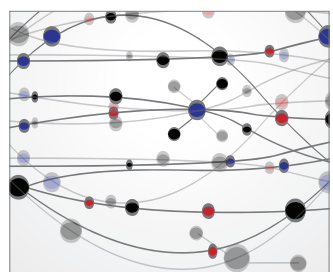

\section{The Scientific} World Journal
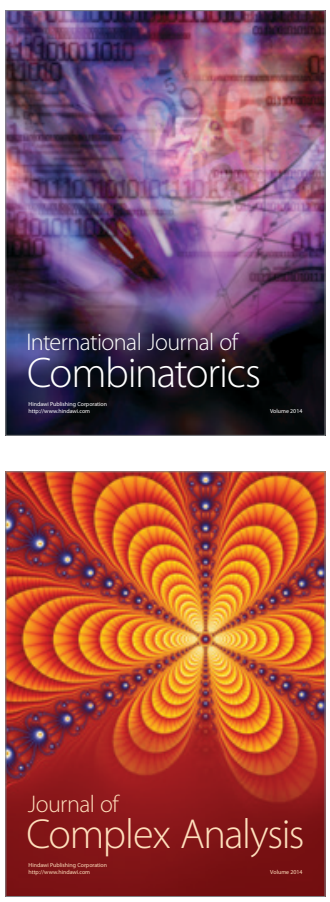

International Journal of

Mathematics and

Mathematical

Sciences
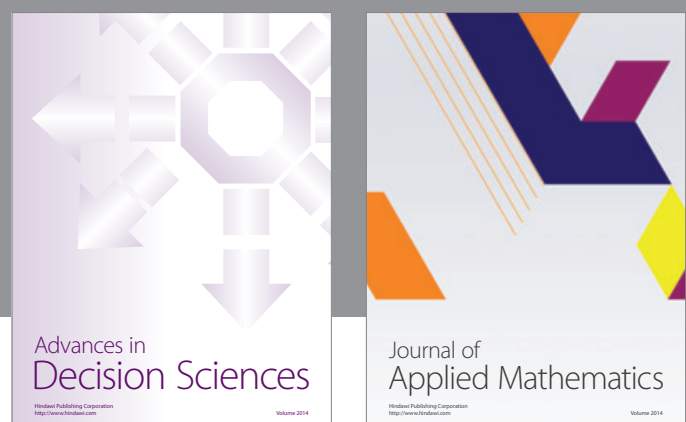

Journal of

Applied Mathematics
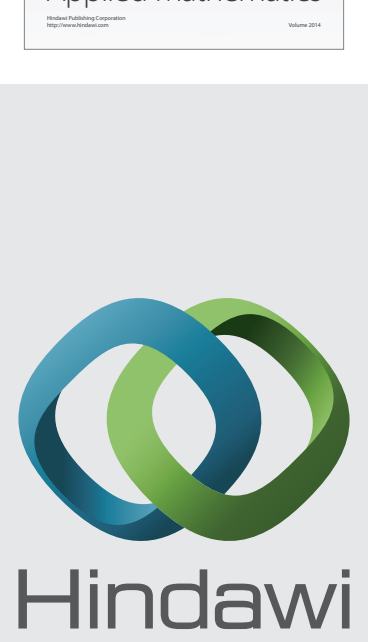

Submit your manuscripts at http://www.hindawi.com
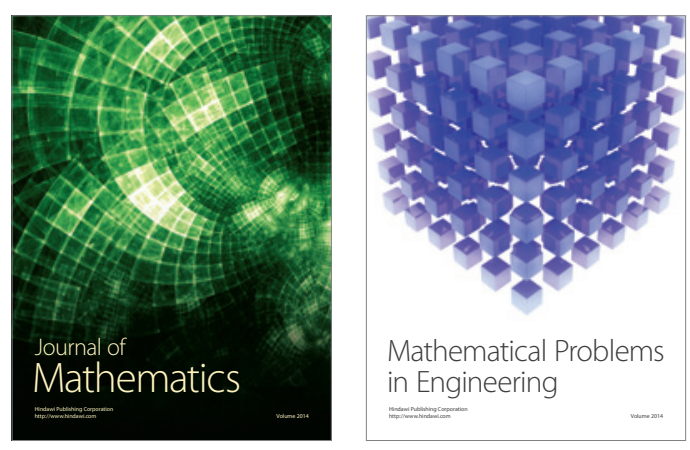

Mathematical Problems in Engineering
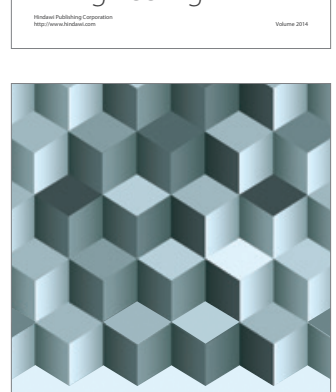

Journal of

Function Spaces
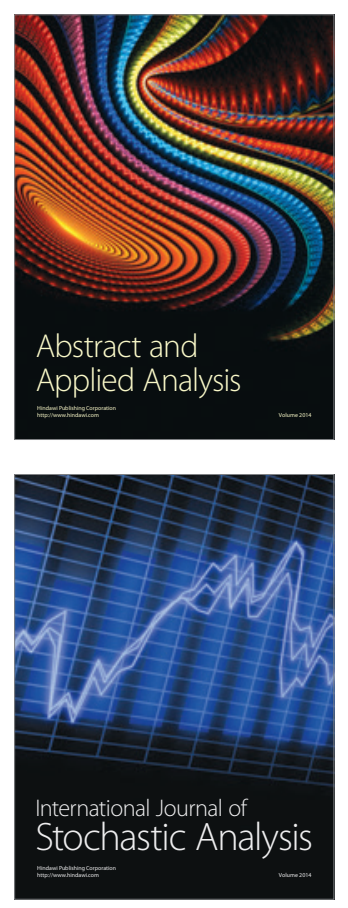

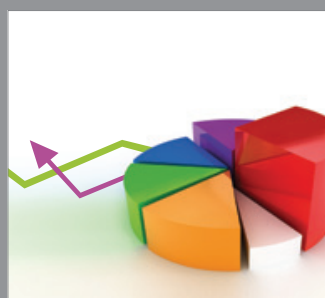

ournal of

Probability and Statistics

Promensencen
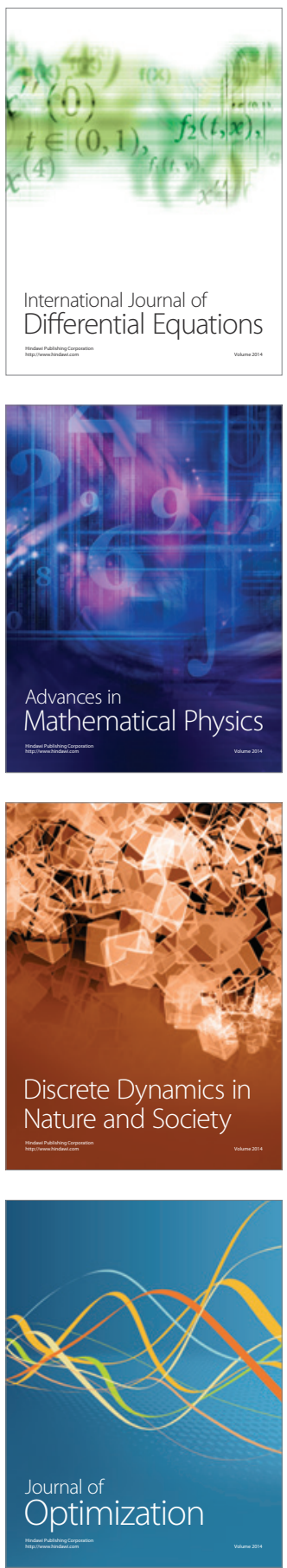